\title{
Ribosomal RNA-depleted RNA sequencing reveals the pathogenesis of refractory Mycoplasma pneumoniae pneumonia in children
}

\author{
FENG HUANG $^{1,2^{*}}$, HUIFENG FAN $^{1 *}$, DIYUAN YANG $^{1}$, JUNSONG ZHANG $^{3}$, \\ TINGTING SHI ${ }^{1}$, DONGWEI ZHANG ${ }^{1}$ and GEN LU ${ }^{1}$ \\ ${ }^{1}$ Department of Respiration, Guangzhou Women and Children's Medical Center, \\ Guangzhou Medical University, Guangzhou, Guangdong 510120; ${ }^{2}$ Institute of Human Virology, \\ Zhongshan School of Medicine, Sun Yat-sen University; ${ }^{3}$ Guangdong Provincial People's Hospital, \\ Guangdong Academy of Medical Sciences, Guangzhou, Guangdong 510080, P.R. China
}

Received April 17, 2020; Accepted May 28, 2021

DOI: $10.3892 / \mathrm{mmr} .2021 .12401$

\begin{abstract}
Pneumonia caused by Mycoplasma pneumoniae (M. pneumoniae) is a major cause of community-acquired pneumonia in children. In some cases, M. pneumoniae pneumonia (MPP) can develop into refractory MPP (RMPP), which shows no clinical or radiological response to macrolides, and can progress to severe and complicated pneumonia. However, the pathogenesis of RMPP remains poorly understood. The present study aimed to identify target genes that could be used as biomarkers for the clinical diagnosis of early-stage RMPP through high-throughput sequencing technology. The differences in long non-coding (lnc)RNAs, mRNAs and circular (circ)RNAs were examined between whole-blood samples from two patients with non-refractory MPP (NRMPP), two patients with RMPP and three healthy children using ribosomal (r)RNA-depleted RNA-sequencing techniques and an integrated mRNA/circRNA analysis. A total of 17 lncRNAs (four upregulated and 13 downregulated), 18 mRNAs (six upregulated and 12 downregulated) and 24 circRNAs (12 upregulated and 12 downregulated) were the most significantly differentially expressed $(\mathrm{P}<0.05)$ between the NRMPP and RMPP groups. Upon functional analysis, the significantly differentially expressed genes encoded by the targeting mRNAs (prostaglandin-endoperoxide synthase 2, IL-8 and fos-like antigen 1) were screened and identified to be enriched in the 'IL-17 signaling pathway'. Furthermore, the key circRNAs
\end{abstract}

Correspondence to: Dr Gen Lu, Department of Respiration, Guangzhou Women and Children's Medical Center, Guangzhou Medical University, 9 Jinsui Road, Zhuijang, Tianhe, Guangzhou, Guangdong 510120, P.R. China

E-mail: lugen5663330@sina.com

*Contributed equally

Key words: refractory Mycoplasma pneumoniae pneumonia, ribosomal RNA-depleted RNA sequencing, long non-coding RNA, circular RNA in the NRMPP and RMPP comparative groups were primarily enriched in 'herpes simplex virus 1 infection', 'viral carcinogenesis' and 'RNA transport'. In the present study, a comprehensive analysis of the differences between the NRMPP and RMPP cases was performed based on rRNA-depleted RNA-sequencing techniques, and the selected genes and circRNAs may be closely associated with the complex pathogenesis of RMPP.

\section{Introduction}

Mycoplasma pneumoniae (M. pneumoniae) is one of the main pathogens that cause respiratory tract infections in humans $(1,2)$. Outbreaks of $M$. pneumoniae pneumonia (MPP) occur in 3- to 7-year cycles worldwide, and epidemics in Korea occurred in 2015 and 2016 (3). M. pneumoniae causes respiratory tract infections in all age groups, accounting for up to $40 \%$ of community-acquired respiratory tract infections in children aged $>5$ years (1). Although MPP is usually a benign and self-limiting process, M. pneumoniae infection can develop into severe, life-threatening diseases, including refractory MPP (RMPP), acute respiratory distress syndrome and necrotizing pneumonitis (2-4). Pneumonia and extrapulmonary complications caused by MPP pose a serious threat to children's health (4). In previous years, MPP has been reported in 10-40\% of cases of community-acquired pneumonia (CAP) and increases during $M$. pneumoniae epidemics $(5,6)$. MPP was previously considered to be a self-limiting process, but RMPP has been reported (7), which shows no clinical or radiological response to macrolides, and may progress to severe pneumonia and cause extrapulmonary complications $(6,7)$. The number of RMPP cases also tends to increase every year; the annual incidence of MPP in respiratory disease was $<10 \%$ of cases of CAP in 2009, $20.5 \%$ in September 2010 and reached a record high of $62.5 \%$ in September 2011 (8). The usual treatment strategy used for MPP is not considered as suitable for RMPP $(7,8)$; thus, an improved understanding of the pathogenesis underlying RMPP is urgently required in order to design a more effective treatment strategy.

Studies on RMPP have indicated that its pathogenesis is primarily associated with the macrolide-resistant mechanism 
of $M$. pneumoniae strains $(9,10)$. Matsuoka et al (11) found that mutations in domains II and V of 23S RNA in M. pneumoniae strains result in a decrease in the affinity of antibiotics to bacterial ribosomes, which eventually leads to resistance to macrolides. In addition, abnormal immune responses caused by MPP (12) and mixed bacterial infections may cause progression of non-refractory MPP (NRMPP) to RMPP (13). However, due to its complexity, the key pathogenesis of RMPP remains unknown.

Long non-coding RNAs (lncRNAs) are >200 nucleotides in length and do not have the capacity to encode proteins (14). The mechanism of action underlying lncRNAs is not completely understood due to their poorly conserved nucleotide sequence. However, previous studies have reported that $\operatorname{lncRNAs}$ regulate gene expression at multiple levels through complex mechanisms $(14,15)$. Circular RNAs (circRNAs) are a novel type of IncRNAs that form a covalently closed continuous loop and thus have no 5'-3' polarity and no polyA tail (16). Accumulating evidence indicates that circRNAs regulate gene expression at both the transcriptional and post-transcriptional levels by serving as microRNA (miRNA) sponges $(16,17)$. circRNAs serve a role in the development of certain diseases, including cancer, diabetes and Alzheimer's disease (16,17-19). Thus, circRNAs may serve as potential biomarkers or therapeutic targets.

The present study performed lncRNA and circRNA profiling via ribosomal (r)RNA-depleted RNA sequencing of whole-blood samples from two patients with NRMPP, two patients with RMPP and three healthy children (HC) to provide a comprehensive analysis of the differences between the $\mathrm{HC}$ and patients with MPP, including the main differences in RNA expression levels between patients with NRMPP and those with RMPP. The present study aimed to identify target genes and circRNAs that may serve as biomarkers for the clinical diagnosis of early-stage disease, as well as providing a theoretical basis for research into the pathogenesis of MPP.

\section{Materials and methods}

Whole-blood sample preparation. The 18 blood samples were collected from December 2018 to December 2019 in the Guangzhou Women and Children's Medical Center(Guangdong, China) from six patients with NRMPP, six patients with RMPP and six healthy children (HC; Table I). Among them, a cohort of seven children (two NRMPP, two RMPP and three HC) was used for high-throughput sequencing, and a cohort of 11 children (four NRMPP, four RMPP and three HC) was used for validation. A blood sample $(2.0 \mathrm{ml})$ was collected from each patient. All patients were tested with the M. pneumoniae-IgM antibody in the serum and M. pneumoniae DNA by PCR in throat swabs on admission, and positive cases were defined as patients with MPP. All patients with MPP were treated with appropriate antibiotics (for example, Azithromycin). Cases with worsening cough, infiltrates on a chest radiograph and a fever that prolonged for $>7$ days were recorded as patients with RMPP. The remaining cases were defined as patients with NRMPP. All specimens were collected at an early stage of MPP on admission ( $\leq 7$ days of onset was defined as an early stage of MPP) (20). The exclusion criteria were as follows: The presence of severe concomitant diseases (chronic pulmonary disease, cardiovascular disease, neoplasia, kidney or liver disease, immune function deficiency and immunodepression); and the presence of mixed infections with other microorganisms. The HC were used as healthy controls.

The present study was approved by the Ethics Committee at Guangzhou Women and Children's Medical Center. The parents or legal guardians of the patients signed written informed consent forms and agreed to its content.

rRNA-depleted RNA sequencing. For rRNA-depleted RNA sequencing, two of the six patients with NRMPP, two of the six patients with RMPP and three of the six HC were screened out as typical cases and sent to Annoroad Gene Technology Co, Ltd. The total RNA of each sample was isolated using TRIzol ${ }^{\circledR}$ reagent (cat. no. MFCD00213058; Invitrogen; Thermo Fisher Scientific, Inc.) according to the manufacturer's protocol. The purity, concentration and integrality of the RNA were determined using a NanoPhotometer ${ }^{\circledR}$ spectrophotometer (IMPLEN), Qubit ${ }^{\circledR}$ 3.0 Fluorometer (Thermo Fisher Scientific, Inc.) and Agilent 2100 RNA Nano 6000 Assay kit (cat. no. 41105500; Agilent Technologies, Inc.), respectively. Subsequently, $3 \mu \mathrm{g}$ RNA from each sample was loaded and the rRNA was removed using Ribo-Zero ${ }^{\text {TM }}$ Gold kits (cat. no. MRZG126; Epicentre; Illumina, Inc.). NEBNext ${ }^{\circledR}$ Ultra $^{\mathrm{TM}}$ Directional RNA Library Prep kit for Illumina ${ }^{\circledR}$ (cat. no. E7420S; New England BioLabs, Inc.) was used to generate the sequencing libraries, which were then sequenced through the Illumina HiSeq platform (Illumina, Inc.). The sequencing type was eukaryotic common transcriptome. The sequencing direction was P5 to P7, then P7 to P5. After removing the low-quality and polluted reads, clean reads were obtained and mapped to the reference genome sequence using Hierarchical Indexing For Spliced Alignment Of Transcripts 2 (version 2.05) (21). The detected reads were mapped to the known mRNA and lncRNA. HTSeq (version 0.6.0) was used to represent the expression level of each gene (22). The loading concentration of the final library was $2 \mathrm{nM}$. DESeq2 Rpackage (version 1.18.0) was used to perform differential expression analysis between the comparative groups (23), and the genes with $\mathrm{P}<0.05$ were considered to be differentially expressed. The unmapped reads were identified as circRNA candidates using Find_circ (version 1.2) and CircRNA Identifier 2 software (version 2.0.1) (23,24). Functional analysis of the differentially expressed genes (DEGs) was performed using Gene Ontology (GO)seq (version 1.0) and Kyoto Encyclopedia of Genes and Genomes (KEGG) Orthology-Based Annotation System (version 2.0) (24-28).

Reverse transcription-quantitative PCR (RT-qPCR). To verify the reliability of the sequencing results, RT-qPCR was performed to determine the expression levels of screened genes (Table SI). EditSeq software (version 7.10, DNASTAR, Inc.) was used to design the specific primers and the $\beta$-actin gene was selected as a standardization control. Briefly, total RNA from blood samples was reverse transcribed into cDNA using the QuantiTect Reverse Transcription kit (Qiagen $\mathrm{GmbH}$ ) according to the manufacturer's instructions. Subsequently, qPCR was performed using a DNA Engine Chromo 4 real-time system (Bio-Rad Laboratories, Inc.) with a TaqMan ${ }^{\mathrm{TM}}$ Copy Number Assay kit (Thermo Fisher Scientific, Inc.). The sequences of the primers are listed in Table II. $\beta$-actin (forward, 5'-GAG GTATCCTGACCCTGAAGTA-3' and reverse, 5'-CACACG CAGCTCATTGTAGA-3') was used as an internal reference. Thermocycling conditions were as follows: $95^{\circ} \mathrm{C}$ for $10 \mathrm{~min}$, 


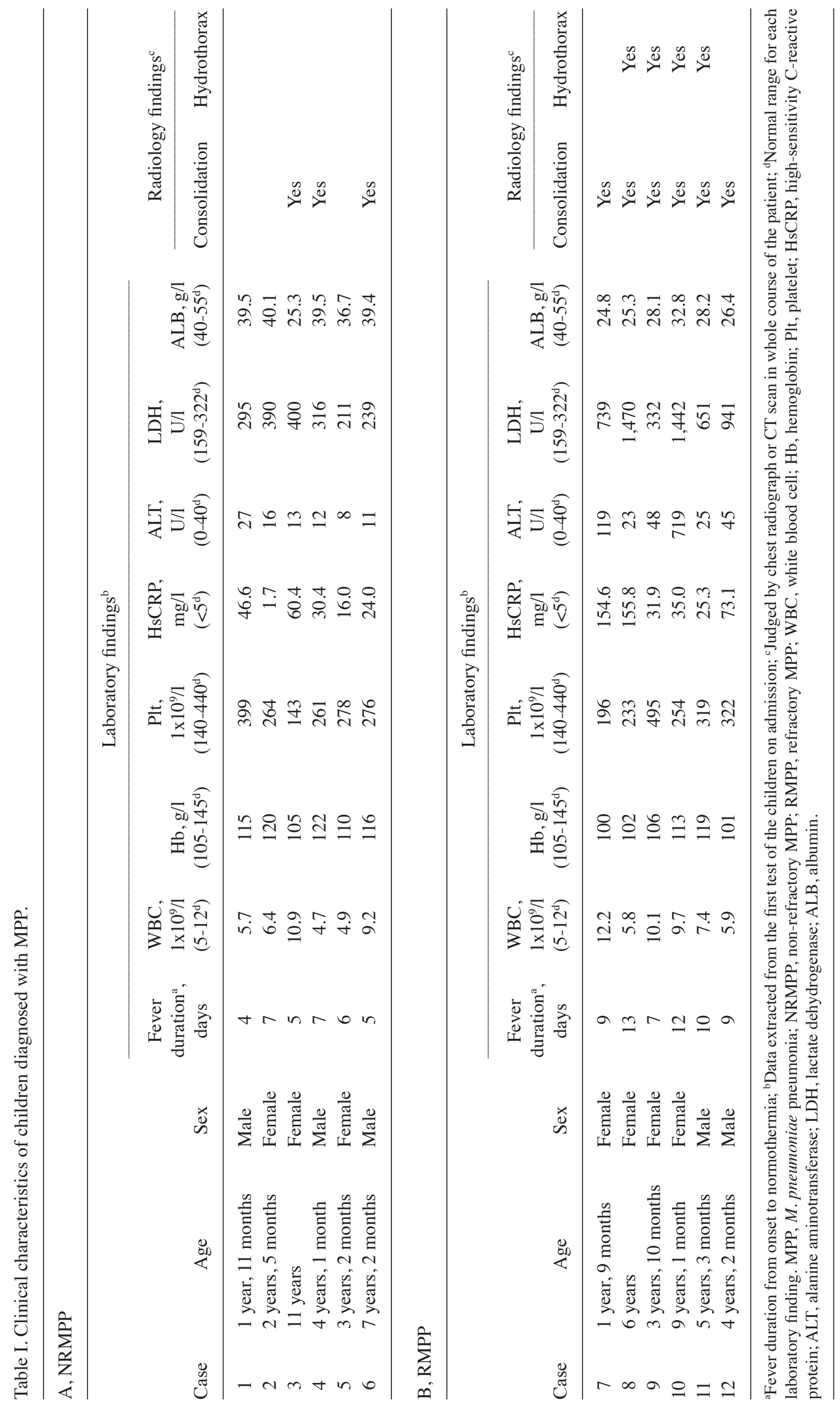




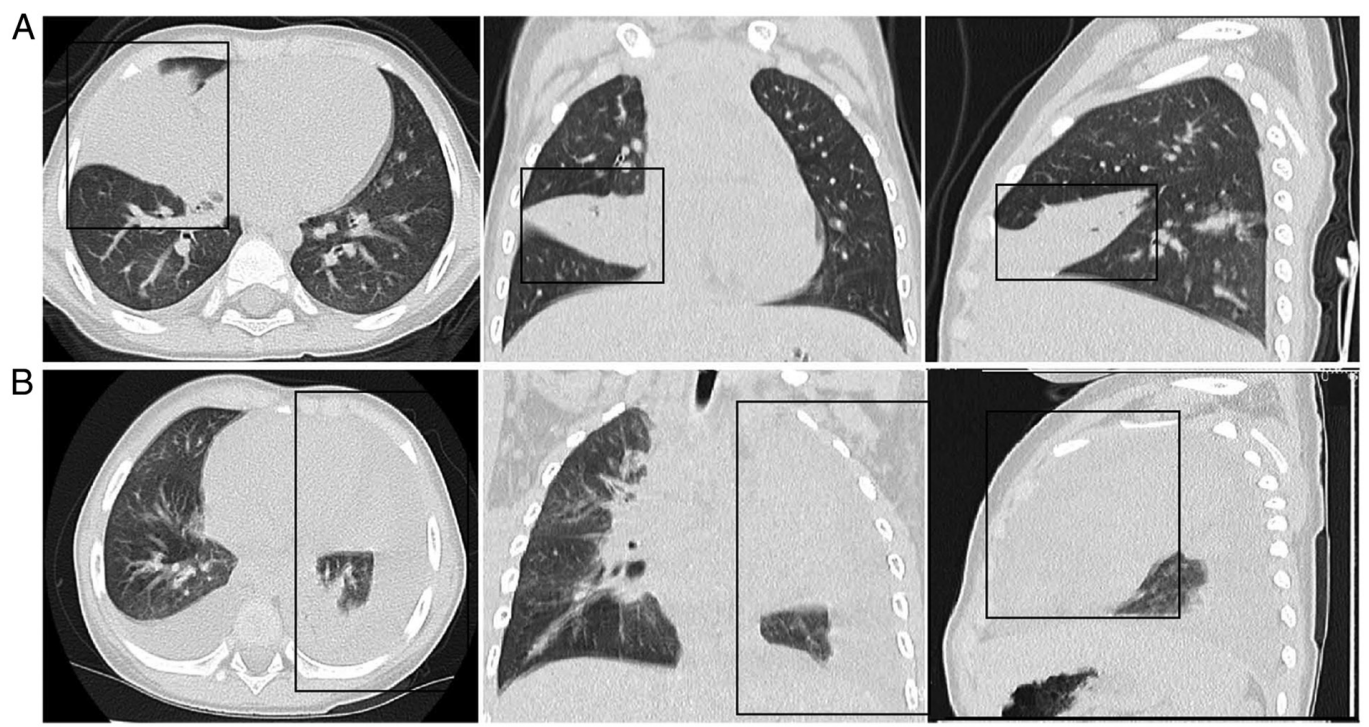

Figure 1. Imaging features of lung injury associated with MPP in children. (A) High-resolution computerized tomography scan of the chest revealing areas of airspace consolidation in the right middle lobe in a 7-year-old child with non-refractory MPP. (B) Areas of airspace consolidation and pleural effusion in the left lobe in a 4-year-old child with refractory MPP. MPP, M. pneumoniae pneumonia.
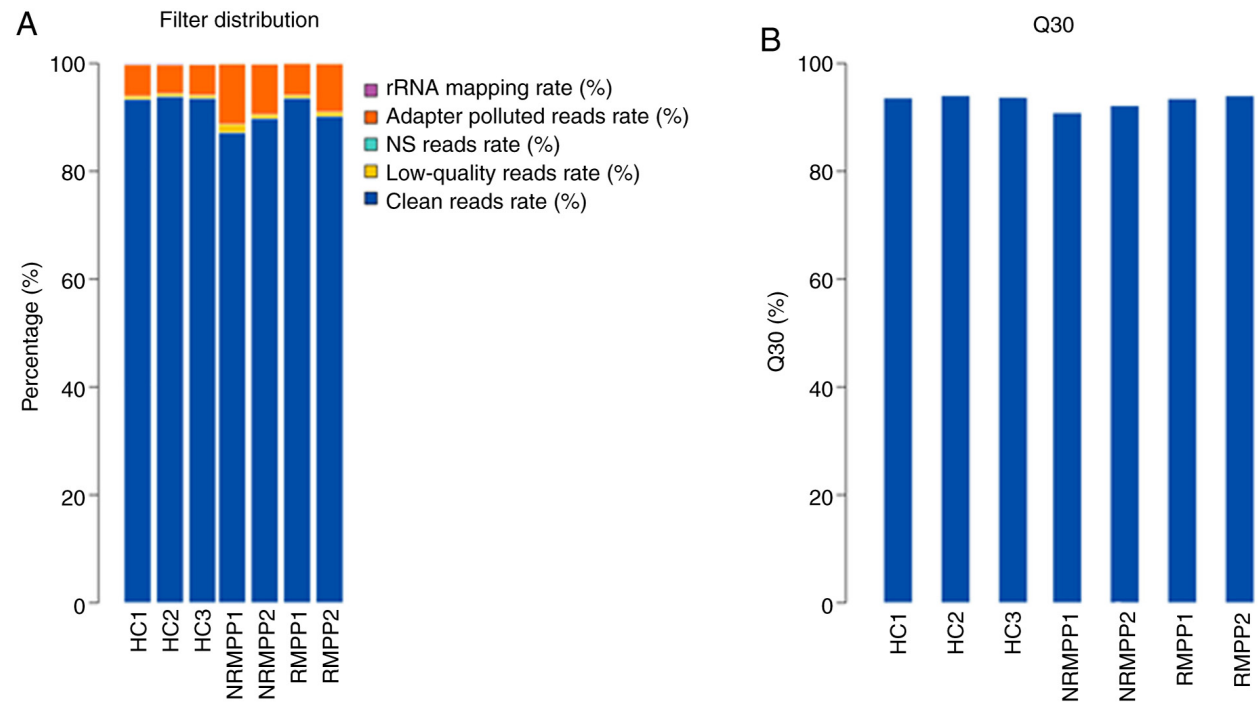

Figure 2. Quality control of rRNA-depleted RNA sequencing. (A) Proportion of various reads before filtration. (B) Q30 ratio (Error rate $<0.1 \%$ ) of sequencing data in each sample. rRNA, ribosomal RNA; NS, sequence-dependent ambiguous bases; MPP, M. pneumoniae pneumonia; NRMPP, non-refractory MPP; RMPP, refractory MPP; HC, healthy children.

followed 40 cycles of $95^{\circ} \mathrm{C}$ for $15 \mathrm{sec}$ and $60^{\circ} \mathrm{C}$ for $\left.60 \mathrm{sec}\right)$. The expression levels were calculated using the $2^{-\Delta \Delta C q}$ method (29).

Statistical analysis. Data were analyzed using GraphPad software (Prism 8.0; GraphPad Software, Inc.) and visualized using the ggplot2 package of R software (version 3.6.1) (30). All data are presented as the mean \pm standard deviation of three independent repeats. An unpaired two-tailed Student's t-test was used to determine the significant differences in IncRNAs, mRNAs and circRNAs between the three groups. For clinical data, the non-parametric Mann-Whitney U test was used for two-group analysis of continuous variables, and the Kruskal-Wallis test followed by Dunn's post hoc test was used for three-group analysis of continuous variables. Fisher's exact test was used for the analysis of categorical variables. P-values are two-sided and were adjusted using the Bonferroni method. $\mathrm{P}<0.05$ was considered to indicate a statistically significant difference.

\section{Results}

Characteristics of patients with MPP. Patients with MPP displayed typical MPP clinical symptoms and were diagnosed with pneumonia. The M. pneumoniae IgM antibody in the serum and M. pneumoniae DNA detected via PCR from throat swabs showed positive results. The clinical characteristics of the cases are listed in Tables SI and SII. Abnormal findings on chest radiographs were observed in all patients with MPP. The chest scans primarily revealed diffuse infiltration of both lungs in NRMPP cases. However, RMPP cases exhibited unequivocal focal or segmental consolidation (Fig. 1A) with pleural effusion (Fig. 1B). 

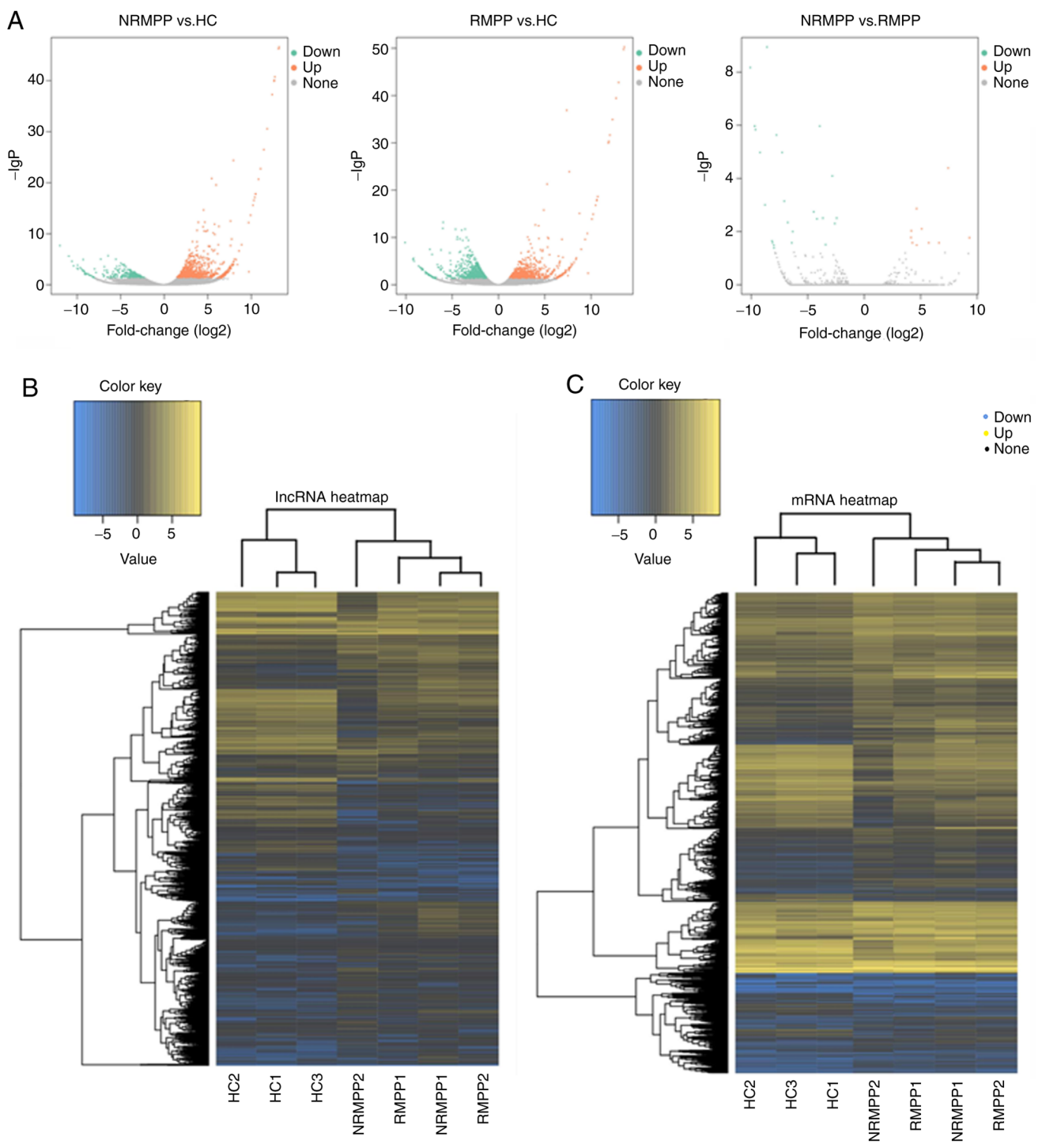

Figure 3. Differential analysis of the DEGs and lncRNAs. (A) Volcano map of DEGs. (B) Cluster map of differentially expressed lncRNAs. (C) Cluster map of differentially expressed mRNAs. Blue represents downregulated, black represents no change and yellow represents upregulated. DEGs, differentially expressed genes; IncRNA, long non-coding RNA; MPP, M. pneumoniae pneumonia; NRMPP, non-refractory MPP; RMPP, refractory MPP; HC, healthy children.

Overview of rRNA-depleted RNA sequencing analysis profiles. Upon rRNA-depleted RNA sequencing, a total of $670,528,498$ raw reads $(102,839,708,98,869,356$ and 93,692,076 for the HC group; 96,341,690 and 92,905,200 for the NRMPP group; and 96,437,832 and 89,442,636 for the RMPP group) were generated. Through filtering the raw data, 614,648,054 clean reads were obtained and mapped to the reference genome (Fig. 2A). The error rate of the filtered data was qualified (Fig. 2B).

A total of 611 lncRNAs (416 upregulated and 195 downregulated) and 692 mRNAs (598 upregulated and 94 downregulated) were significantly differentially expressed $(\mathrm{P}<0.05)$ between the NRMPP and $\mathrm{HC}$ groups (Fig. 3). A total of 937 lncRNAs (433 upregulated and 504 downregulated) and 1,027 mRNAs (593 upregulated and 434 downregulated) were significantly differentially expressed $(\mathrm{P}<0.05)$ between the RMPP and HC groups. A total of 17 lncRNAs (4 upregulated and 13 downregulated) and 18 mRNAs (6 upregulated and 12 downregulated) were significantly differentially expressed
$(\mathrm{P}<0.05)$ between the RMPP and NRMPP groups (Table III). The significantly differentially expressed lncRNAs between the RMPP and NRMPP groups included ENSG00000249790, ENSG00000261026, MSTRG.215206, MSTRG.233743, MSTRG.238033, MSTRG.238419, MSTRG.268000, MSTRG.275241 (Table III).

Bioinformatics analysis of sequencing profiles. The differentially expressed mRNAs between the RMPP and NRMPP groups (Table IV) were ENSG00000073756, ENSG00000111788, ENSG00000122877, ENSG00000123838, ENSG00000130656, ENSG00000165949; these were identified by functional analysis using both the GO and KEGG databases. In the GO analysis, the significantly differentially expressed mRNAs were primarily enriched in 'complement activation, classical pathway', 'leukocyte migration' and 'chemotaxis' (Fig. 4A). In the KEGG pathway analysis, the significantly differentially expressed mRNAs were primarily enriched in the 'IL-17 signaling pathway' (Fig. 4B). RT-qPCR was used to verify candidate 
Table II. Primers used for reverse transcription-quantitative PCR.

\begin{tabular}{ll}
\hline circRNA & \\
\hline hsa_circ_0022808 & Primers $\left(5^{\prime} \rightarrow 3^{\prime}\right)$ \\
hsa_circ_0006793 & R: GAGGAAGAGGATGCAGGAGC \\
hsa_circ_0014390 & F: AGTCCCCTGCTATCACTGGT \\
hsa_circ_0014305 & R: CCAGCTTCGGTCACTGAACA \\
hsa_circ_00216400 & F: TCTGTTGAAGATTTGAAGAACCCA \\
& R: CTGAGGGCTAGAGGACTGGT \\
& F: TTCTCCCTGGCGGAGGAATA \\
& R: GGATGGCTGGTTTGAAGCAC \\
& F: CTTCAGTACCAGAGCCCCAC \\
& R: ACCACCTCAACCGTTTCAACT
\end{tabular}

F, forward; R, reverse; circ, circular RNA.

Tables III. Significantly differentially expressed long non-coding RNAs between RMPP and NRMPP groups.

\begin{tabular}{|c|c|c|c|c|c|}
\hline \multirow[b]{2}{*}{ Gene } & \multicolumn{2}{|c|}{$\begin{array}{c}\text { Relative gene expression } \\
\text { levels }\end{array}$} & \multirow[b]{2}{*}{ P-value } & \multirow[b]{2}{*}{ Up/downregulated } & \multirow[b]{2}{*}{ Position } \\
\hline & RMPP & NRMPP & & & \\
\hline ENSG00000249790 & 635.1540951 & 3447.840859 & $3.09 \times 10^{-3}$ & Down & chr12:8788257-8795789:+ \\
\hline ENSG00000261026 & 472.7296308 & 4.781416405 & $2.65 \times 10^{-2}$ & Up & chr8:22679013-22684009:- \\
\hline MSTRG.215206 & 2391.068595 & 70.25707389 & $7.87 \times 10^{-3}$ & $\mathrm{Up}$ & chr15:48211935-48296341:- \\
\hline MSTRG.233743 & 63.78915178 & 0 & $1.70 \times 10^{-2}$ & $\mathrm{Up}$ & chr16:15028952-15030258:- \\
\hline MSTRG.238033 & 0 & 27.68733409 & $2.65 \times 10^{-2}$ & Down & chr16:34582662-34587722:+ \\
\hline MSTRG.238419 & 0.404276272 & 87.94800239 & $2.34 \times 10^{-6}$ & Down & chr16:46388508-46406972:+ \\
\hline MSTRG.268000 & 5.600197692 & 101.7282093 & $3.32 \times 10^{-3}$ & Down & chr17:74562930-74563656:- \\
\hline MSTRG.275241 & 0 & 25.47228864 & $3.27 \times 10^{-2}$ & Down & chr18:22348684-22349256:- \\
\hline MSTRG.299858 & 0.404276272 & 34.38883192 & $2.65 \times 10^{-2}$ & Down & chr19:37282731-37293921:- \\
\hline MSTRG.32896 & 0.404276272 & 54.56033482 & $7.16 \times 10^{-4}$ & Down & chr1:143501630-143505359:+ \\
\hline MSTRG.384208 & 0 & 83.06200226 & $1.08 \times 10^{-6}$ & Down & chr21:8219668-8220285:- \\
\hline MSTRG.384231 & 0 & 78.99033548 & $1.45 \times 10^{-6}$ & Down & chr21:8446933-8448138:- \\
\hline MSTRG.386206 & 0 & 28.50166744 & $2.23 \times 10^{-2}$ & Down & chr21:22451534-22451867:+ \\
\hline MSTRG.488193 & 0 & 25.24433402 & $4.13 \times 10^{-2}$ & Down & chr4:146285918-146287333:+ \\
\hline MSTRG.631597 & 321.397898 & 16.70145272 & $2.65 \times 10^{-2}$ & Up & chr8:32733246-32739937:+ \\
\hline MSTRG.678280 & 0.490427811 & 39.44287588 & $9.98 \times 10^{-3}$ & Down & chr9:101736669-101848060:+ \\
\hline
\end{tabular}

MPP, M. pneumoniae pneumonia; NRMPP, non-refractory MPP; RMPP, refractory MPP.

genes that may be involved in pathogenesis of RMPP, such as prostaglandin-endoperoxide synthase 2 (PTGS2), chemokine (C-X-C motif) ligand 8 (CXCL8) and Fos-like antigen 1 (FOSL1; Fig. 4C) and the primers were designed by EditSeq software.

In the circRNA/miRNA co-expression analysis, a total of 1,370 circRNAs (505 upregulated and 865 downregulated) were significantly differentially expressed $(\mathrm{P}<0.05)$ between the HC and MPP groups (Fig. 5A and B). The functions of circRNAs were associated with the known function of the host linear transcripts and annotated by the GO and KEGG databases. In the GO analysis of the host linear transcripts, the differentially expressed terms were classified into three categories. Under biological processes, the GO terms were primarily enriched in 'regulation of mRNA metabolic process', 'nucleobase-containing compound transport' and 'RNA localization' (Fig. 5D). Under the category of cellular component, the GO terms were primarily enriched in 'nuclear speck', 'cell-substrate junction' and 'focal adhesion' (Fig. 5E). Under the category of molecular function, the GO terms were primarily enriched in 'ubiquitin-like protein transferase activity', 'ribonucleoprotein complex binding' and 'enhancer binding' (Fig. 5F). In the KEGG pathway analysis, the significant DEGs were primarily enriched in 'Herpes simplex virus 1 infection', 'viral carcinogenesis' and 'RNA 

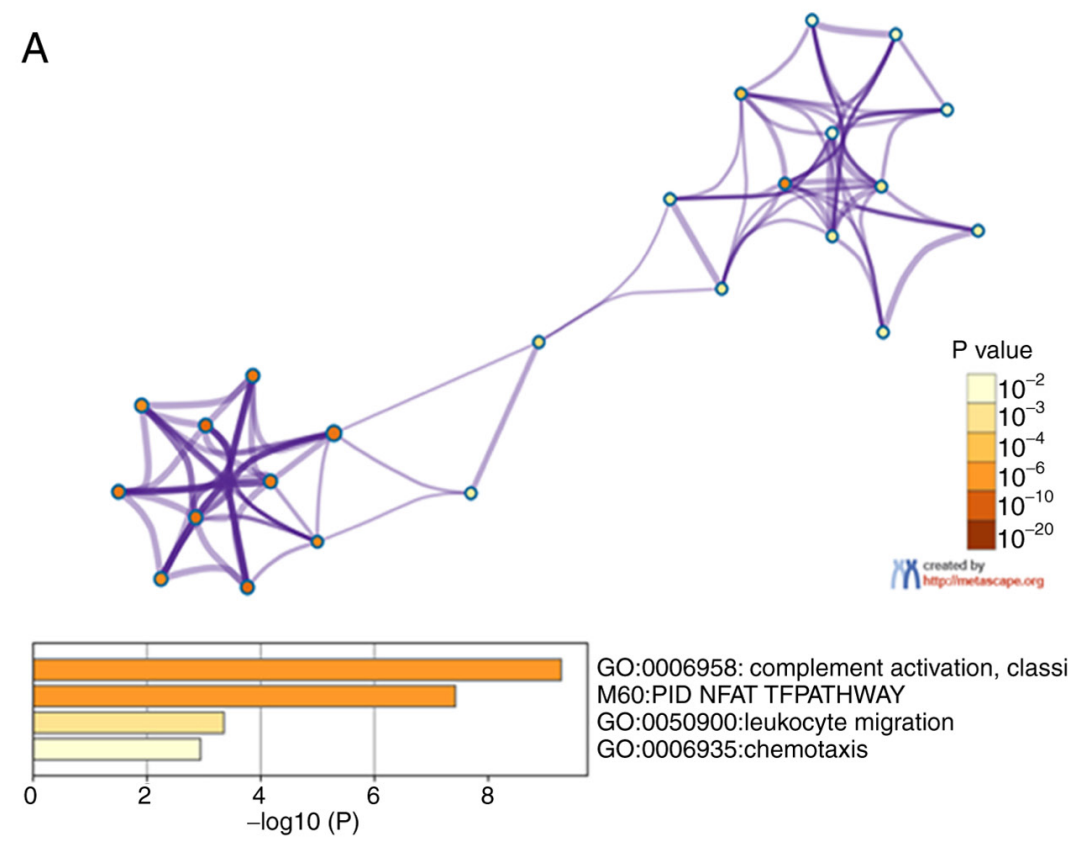

GO:0006958: complement activation, classical pathway M60:PID NFAT TFPATHWAY GO:0050900:leukocyte migration GO:0006935:chemotaxis

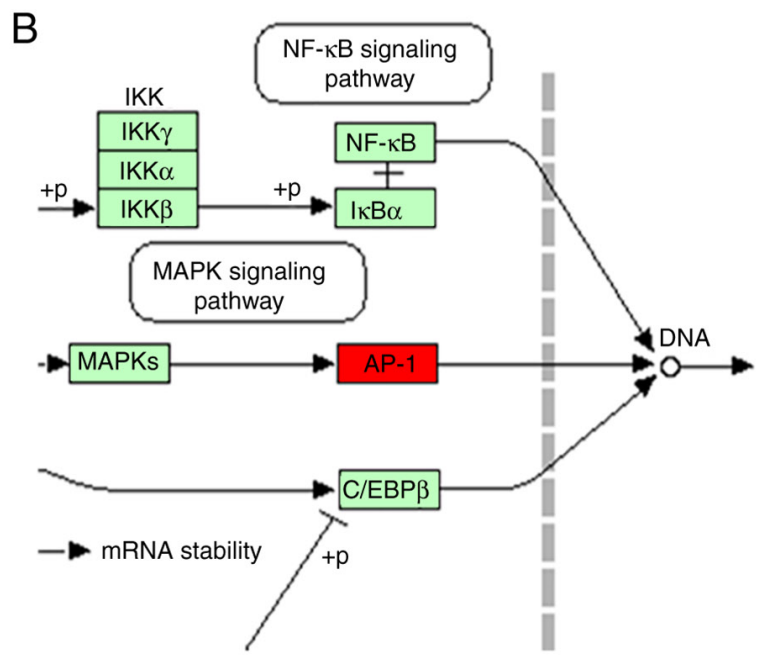

Chemokins

\begin{tabular}{|c|c|c|c|}
\hline CXCL1 & CXCL2 & CXCL5 & CXCL8 \\
\hline CXCL10 & CCL2 & CCL7 & CCL20 \\
\hline
\end{tabular}

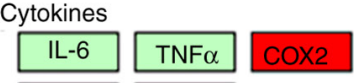

G-CSF GM-CSR

Anti-microbial

defensin MUC5Ad MUC5B

S100A7 S100A8 S100A9 LCN2

Tissue remodeling

\begin{tabular}{|l|l|}
\hline MMP1 MMP3 MMP9 MMP13 \\
\hline
\end{tabular}

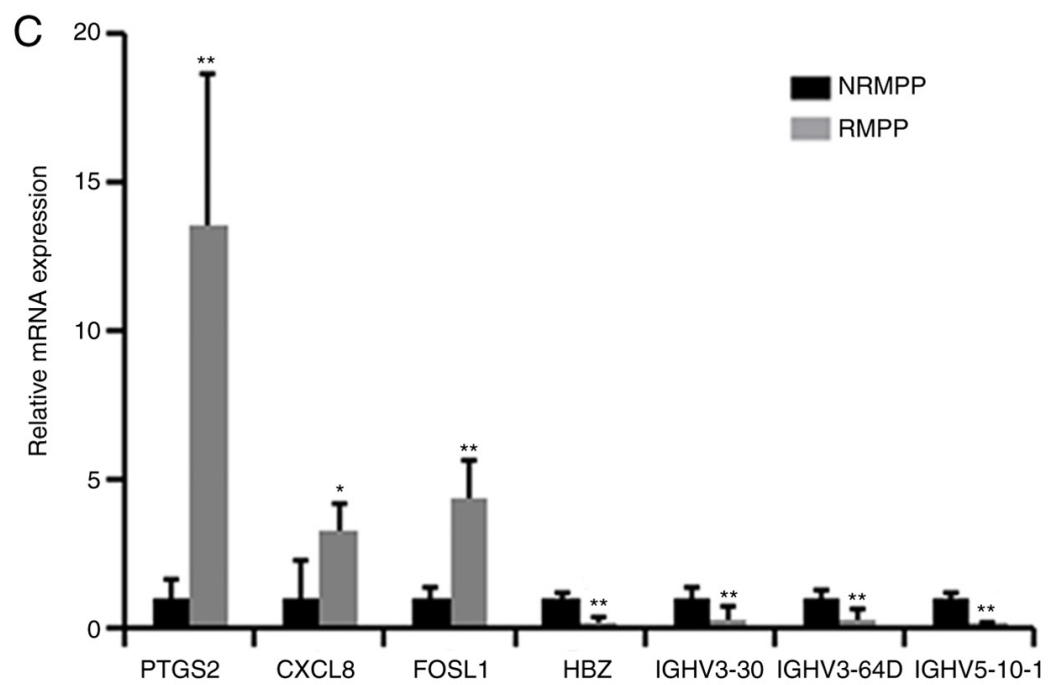

Figure 4. Functional analysis of the DEGs between the RMPP and NRMPP groups. (A) GO analysis of the DEGs. (B) DEGs enriched in the 'IL-17 signaling pathway'. (C) Validation of DEGs via reverse transcription-quantitative PCR. For the presented data, four NRMPP, four RMPP and three HC were used. Comparisons between two groups were analyzed using Student's t-test. " $\mathrm{P}<0.05$ and ${ }^{* *} \mathrm{P}<0.01$ vs. NRMPP group. DEGs, differentially expressed genes; GO, Gene Ontology; MPP, M. pneumoniae pneumonia; NRMPP, non-refractory MPP; RMPP, refractory MPP; HC, healthy children; MPPS, severe MPP; MPPM, mild MPP; PTGS2, prostaglandin-endoperoxide synthase 2; CXCL8, interleukin 8; FOSL1, fos-like antigen 1; HBZ, hemoglobin subunit $\zeta$; IGHV, immunoglobulin heavy variable. 
Table IV. Significantly differentially expressed mRNAs between RMPP and NRMPP groups.

Relative gene expression levels

\begin{tabular}{|c|c|c|c|c|c|c|}
\hline Gene & RMPP & NRMPP & P-value & $\begin{array}{c}\mathrm{Up} / \\
\text { downregulated }\end{array}$ & Gene name & Description \\
\hline ENSG00000073756 & 9780.85878 & 553.0410947 & $1.67 \times 10^{-2}$ & Up & PTGS2 & $\begin{array}{l}\text { Prostaglandin-endoperoxide } \\
\text { synthase } 2\end{array}$ \\
\hline ENSG00000111788 & 9.669441835 & 103.3909011 & $3.10 \times 10^{-2}$ & Down & DDX11L8 & $\begin{array}{l}\text { Putative ATP-dependent RNA } \\
\text { helicase DDX11-like protein } 8\end{array}$ \\
\hline ENSG00000122877 & 536.8492789 & 21.50520559 & $1.37 \times 10^{-3}$ & Up & EGR2 & Early growth response protein 2 \\
\hline ENSG00000123838 & 240.3692975 & 4.57224895 & $2.65 \times 10^{-2}$ & Up & C4BPA & $\begin{array}{l}\text { Complement component } 4 \\
\text { binding protein } \alpha\end{array}$ \\
\hline ENSG00000130656 & 0 & 43.9105411 & $9.88 \times 10^{-4}$ & Down & HBZ & Hemoglobin subunit $\zeta$ \\
\hline ENSG00000165949 & 23.52076078 & 359.2170269 & $1.08 \times 10^{-6}$ & Down & IFI27 & $\begin{array}{l}\text { Interferon } \alpha \text {-inducible protein } 27 \\
\text { mitochondrial }\end{array}$ \\
\hline ENSG00000169429 & 5129.9448 & 284.2008731 & $9.35 \times 10^{-3}$ & Up & CXCL8 & Interleukin 8 \\
\hline ENSG00000175592 & 137.6919176 & 5.491166033 & $3.22 \times 10^{-2}$ & Up & FOSL1 & Fos-like antigen 1 \\
\hline ENSG00000185736 & 0.490427811 & 190.8606578 & $1.14 \times 10^{-9}$ & Down & ADARB2 & $\begin{array}{l}\text { Double stranded RNA-specific } \\
\text { editase B }\end{array}$ \\
\hline ENSG00000188056 & 0.490427811 & 75.33804428 & $1.06 \times 10^{-5}$ & Down & TREML4 & Trem-like transcript 4 protein \\
\hline ENSG00000211653 & 82.7834293 & 501.0131059 & $5.00 \times 10^{-3}$ & Down & IGLV1-40 & $\begin{array}{l}\text { Immunoglobulin lambda variable } \\
1-40\end{array}$ \\
\hline ENSG00000211897 & 570.423636 & 4031.523518 & $8.08 \times 10^{-5}$ & Down & IGHG3 & $\begin{array}{l}\text { Immunoglobulin heavy constant } \\
\gamma 3\end{array}$ \\
\hline ENSG00000236770 & 0.404276272 & 43.86941747 & $4.53 \times 10^{-3}$ & Down & CD300C & CMRF35-like molecule 6 \\
\hline ENSG00000237973 & 1159.431015 & 6.701497833 & $4.07 \times 10^{-5}$ & Up & COX1 & Cytochrome c oxidase subunit 1 \\
\hline ENSG00000270550 & 3.664967871 & 81.06781283 & $1.81 \times 10^{-3}$ & Down & IGHV3-30 & $\begin{array}{l}\text { Immunoglobulin heavy variable } \\
3-30\end{array}$ \\
\hline ENSG00000282639 & 0 & 59.68597813 & $1.06 \times 10^{-5}$ & Down & IGHV3-64D & $\begin{array}{l}\text { Immunoglobulin heavy variable } \\
\text { 3-64D }\end{array}$ \\
\hline ENSG00000282651 & 0 & 108.602341 & $6.73 \times 10^{-9}$ & Down & IGHV5-10-1 & $\begin{array}{l}\text { Immunoglobulin heavy variable } \\
5-10-1\end{array}$ \\
\hline ENSG00000284690 & 43.90687745 & 553.5974251 & $3.09 \times 10^{-3}$ & Down & $\mathrm{CD} 300 \mathrm{H}$ & Protein $\mathrm{CD} 300 \mathrm{H}$ \\
\hline
\end{tabular}

MPP, M. pneumoniae pneumonia; NRMPP, non-refractory MPP; RMPP, refractory MPP.

transport' (Fig. 5C). The top 11 significantly differentially expressed circRNAs between the HC and MPP groups are listed in Table V.

A total of 156 circRNAs (85 upregulated and 71 downregulated) were significantly differentially expressed $(\mathrm{P}<0.05)$ between the NRMPP and RMPP groups (Fig. 6A-C). A total of 24 circRNAs were identified as the most significantly differentially expressed circRNAs between the NRMPP and RMPP groups. In the GO analysis, GO terms were primarily enriched in 'positive regulation of myeloid cell differentiation' and 'positive regulation of hemopoiesis' (Fig. 6D). The screened circRNAs (Table VI) were primarily enriched in 'colorectal cancer', 'hepatitis B' and 'apoptosis' (Fig. 6E). The top five upregulated circRNAs were selected for further validation by performing RT-qPCR (Fig. 6F), indicating that these circRNAs may serve as potential biomarkers for RMPP.

\section{Discussion}

Although the macrolide-resistant mechanisms of M.pneumoniae strains and excessive immunological inflammation are the most commonly proposed mechanisms underlying RMPP $(3,4)$, the pathogenesis of RMPP remains to be elucidated and there is still a lack of accurate assessment tools and biomarkers for RMPP. At present, the common methods for estimating the severity of RMPP are based on clinical characteristics, pulmonary imaging severity and therapeutic effect, which are unable to ensure an effective identification of early-stage RMPP $(5,6)$. Therefore, it is necessary to identify novel tools and biomarkers for the early 
A HC_vs_MPP (Pvalue $=0.05, \log 2 \mathrm{FC}=2)$

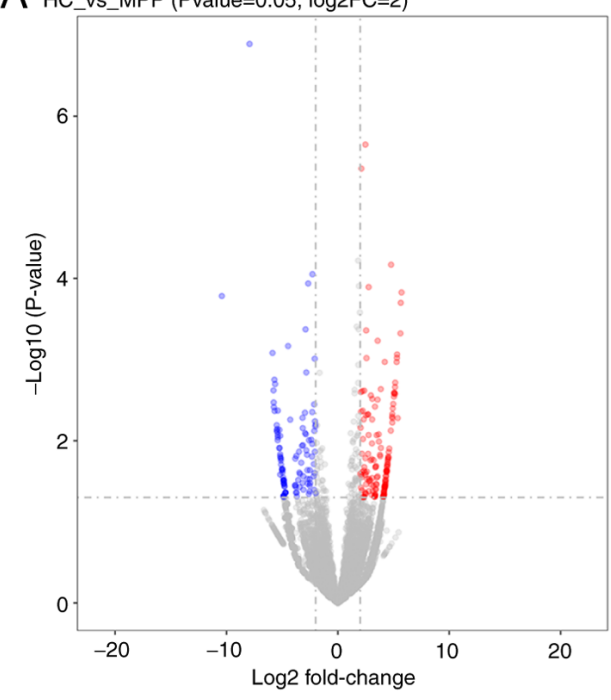

C
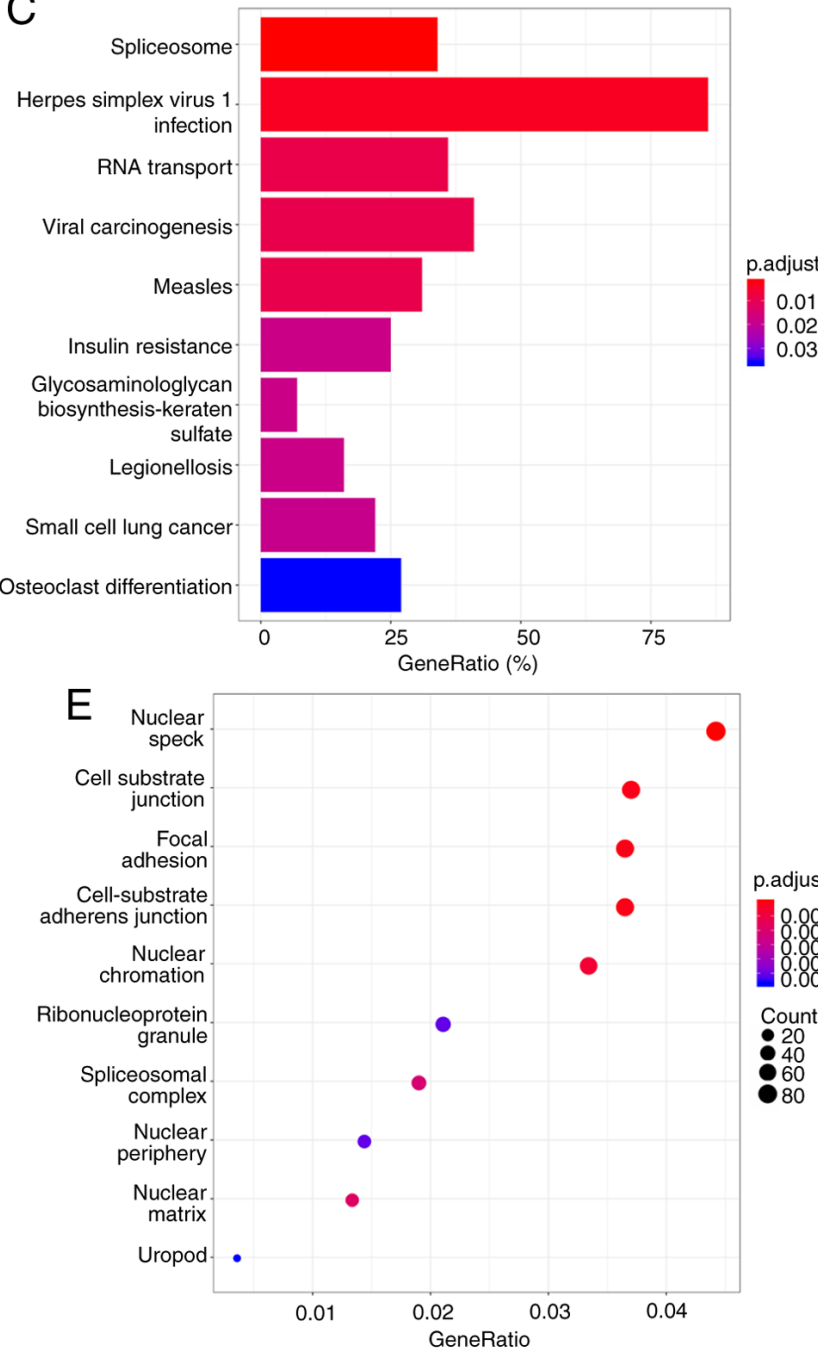

B
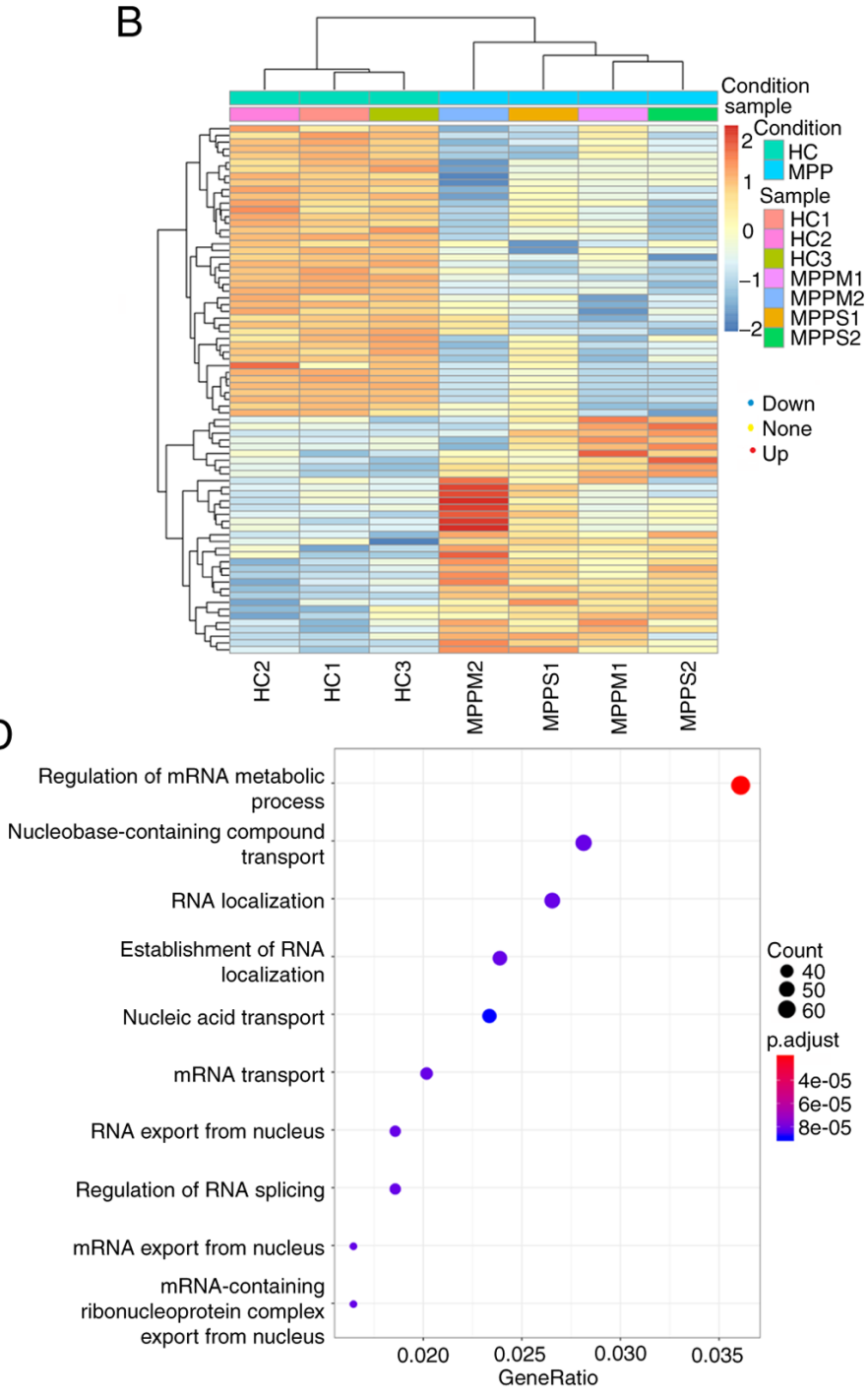

$\mathrm{F}$

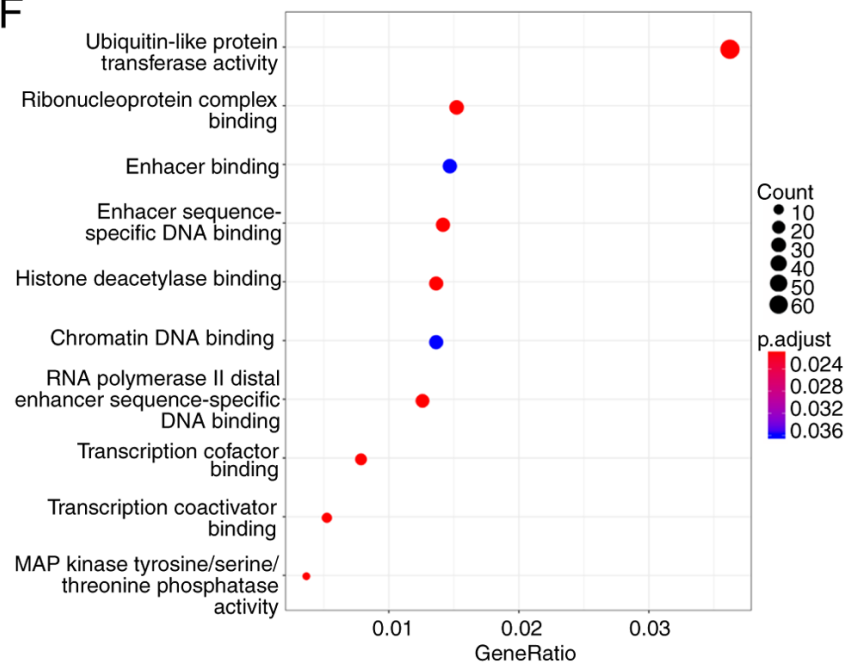

Figure 5. Functional analysis of the circRNAs between the HC and MPP groups. (A) Volcano plot and (B) cluster map of significantly differentially expressed circRNAs between the HC and MPP groups. Red represents upregulated, blue represents downregulated and yellow represents no change. (C) The most enriched pathways between the HC and MPP groups. The most enriched Gene Ontology terms classified into three main categories: (D) Biological processes, (E) cellular component and (F) molecular function. circRNA, circular RNA; HC, healthy children; MPP, M. pneumoniae pneumonia; MPPS, severe MPP; MPPM, mild MPP.

diagnosis of RMPP. The present study was designed to identify target genes implicated in the pathogenesis of RMPP to enable early diagnosis by comparing the differences between the cases with NRMPP and those with RMPP. To the best of our knowledge, the present study was the first to assess the differences in lncRNAs and circRNAs between NRMPP and RMPP. 
Table V. Significantly differentially expressed circRNAs between HC and MPP groups.

\begin{tabular}{|c|c|c|c|c|c|}
\hline Gene & Log2FoldChange & P-value & Best transcript & Gene symbol & $\begin{array}{l}\text { Upregulated/ } \\
\text { downregulated }\end{array}$ \\
\hline hsa_circ_0002171 & 10.42052522 & 0.029534 & NM_198567 & C5orf25 & Up \\
\hline hsa_circ_0001535 & 7.929259862 & 0.0002309 & NM_001101801 & FAM13B & Up \\
\hline hsa_circ_0007261 & 2.669813966 & 0.0254796 & NM_031921 & ATAD3B & Up \\
\hline hsa_circ_0018432 & 2.28731687 & 0.0254796 & NM_194298 & SLC16A9 & Up \\
\hline hsa_circ_0014535 & -1.810846752 & 0.0242991 & NM_002004 & FDPS & Down \\
\hline hsa_circ_0002316 & -1.891464967 & 0.0254796 & NM_145243 & OMA1 & Down \\
\hline hsa_circ_0004327 & -1.973037708 & 0.0429052 & NM_007124 & UTRN & Down \\
\hline hsa_circ_0019868 & -2.107107635 & 0.0026471 & NM_014631 & SH3PXD2A & Down \\
\hline hsa_circ_0001726 & -2.465790354 & 0.002007 & NM_032164 & ZNF394 & Down \\
\hline hsa_circ_0026176 & -2.760620461 & 0.0254796 & NM_003217 & TMBIM6 & Down \\
\hline hsa_circ_0021682 & -4.772727352 & 0.0242991 & NM_024662 & NAT10 & Down \\
\hline
\end{tabular}

MPP, M. pneumoniae pneumonia; HC, healthy children; circRNA, circular RNA.
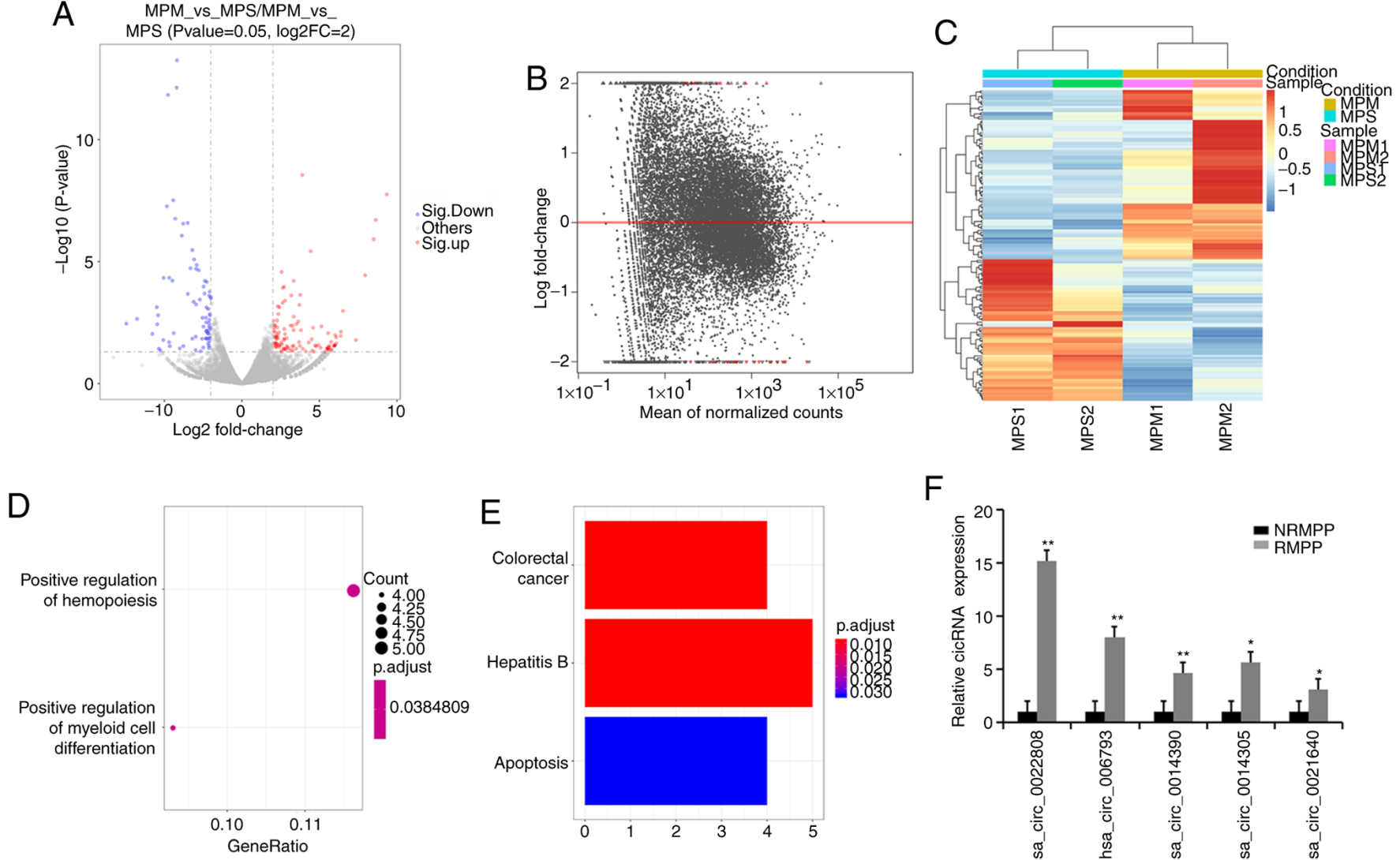

$\mathrm{F}$

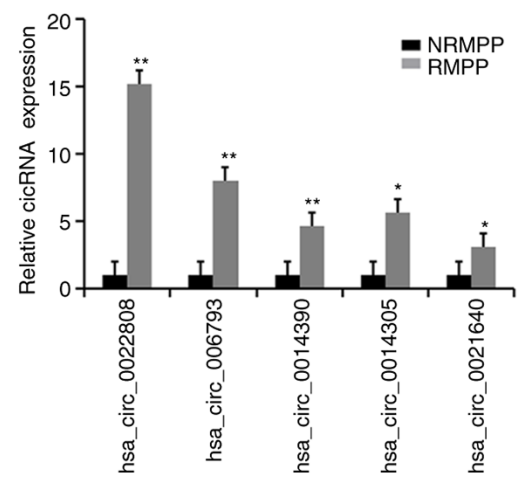

Figure 6. Functional analysis of the circRNAs between the NRMPP and RMPP groups. (A) Volcano plot, (B) Lateral view of the volcano plot and (C) cluster map of significantly differentially expressed circRNAs between the NRMPP and RMPP groups. (D) Most enriched Gene Ontology terms. (E) Most enriched Kyoto Encyclopedia of Genes and Genomes pathways between the NRMPP and RMPP groups. (F) Validation of the expression of the selected circRNAs via reverse transcription-quantitative PCR. For the presented data, four NRMPP, four RMPP and three HC were used. Comparisons between two groups were analyzed using Student's t-test. ${ }^{*}<<0.05$ and ${ }^{* *} \mathrm{P}<0.01$ vs. NRMPP group. circRNA, circular RNA; MPP, M. pneumoniae pneumonia; NRMPP, non-refractory MPP; RMPP, refractory MPP; HC, healthy children; MPPS, severe MPP; MPPM, mild MPP.

circRNAs serve important roles in regulating gene expression by sequestering miRNAs as a sponge at the transcriptional or post-transcriptional levels (16). Thus, circRNAs can regulate a number of processes associated with numerous diseases, such as cancer $(16,17)$. M. pneumoniae possesses a tip-like organelle that permits a highly oriented extracellular parasitism of the respiratory epithelium to avoid clearance by mucosal cilia and phagocytosis, and its adhesion ability is positively correlated 
Table VI. Significantly differentially expressed circRNAs between RMPP and NRMPP groups.

\begin{tabular}{|c|c|c|c|c|c|}
\hline Gene & Log2FoldChange & P-value & Best transcript & Gene symbol & $\begin{array}{l}\text { Upregulated/ } \\
\text { downregulated }\end{array}$ \\
\hline hsa_circ_0022808 & 23.10152809 & $1.38 \times 10^{-6}$ & NM_002689 & POLA2 & Up \\
\hline hsa_circ_0006793 & 7.812895001 & $4.88 \times 10^{-2}$ & NM_001242767 & MTHFD1L & Up \\
\hline hsa_circ_0014390 & 6.98745233 & $3.28 \times 10^{-3}$ & NM_014847 & UBAP2L & Up \\
\hline hsa_circ_0014305 & 6.373176867 & $9.65 \times 10^{-3}$ & NM_023015 & INTS3 & Up \\
\hline hsa_circ_0021640 & 6.166346606 & $1.54 \times 10^{-2}$ & NM_005898 & CAPRIN1 & Up \\
\hline hsa_circ_0017689 & 6.1204205 & $1.71 \times 10^{-2}$ & NM_014688 & USP6NL & Up \\
\hline hsa_circ_0025915 & 6.067184847 & $1.80 \times 10^{-2}$ & NM_025003 & ADAMTS20 & Up \\
\hline hsa_circ_0003781 & 5.86144273 & $12.85 \times 10^{-2}$ & NM_001242865 & PRMT2 & Up \\
\hline hsa_circ_0024849 & 5.834493906 & $2.66 \times 10^{-2}$ & NM_001143835 & NFRKB & Up \\
\hline hsa_circ_0015519 & 5.737917011 & $3.35 \times 10^{-2}$ & NM_004736 & XPR1 & Up \\
\hline hsa_circ_0005901 & 5.506362304 & $4.75 \times 10^{-2}$ & TCONS_00024174 & TCONS_00024174 & Up \\
\hline hsa_circ_0023925 & 5.506362304 & $4.75 \times 10^{-2}$ & NM_007166 & PICALM & Up \\
\hline hsa_circ_0025400 & -4.680048526 & $3.23 \times 10^{-2}$ & NM_004426 & PHC1 & Down \\
\hline hsa_circ_0010030 & -5.606173248 & $4.84 \times 10^{-2}$ & NM_201628 & KAZN & Down \\
\hline hsa_circ_0011249 & -5.645431582 & $4.67 \times 10^{-2}$ & NM_001020658 & PUM1 & Down \\
\hline hsa_circ_0004477 & -5.753705882 & $3.74 \times 10^{-2}$ & NM_182751 & MCM10 & Down \\
\hline hsa_circ_0025209 & -5.791153915 & $3.88 \times 10^{-2}$ & NM_001033714 & NOP2 & Down \\
\hline hsa_circ_0018110 & -5.819081289 & $3.37 \times 10^{-2}$ & NM_004521 & KIF5B & Down \\
\hline hsa_circ_0010131 & -5.889114841 & $3.05 \times 10^{-2}$ & NM_004431 & EPHA2 & Down \\
\hline hsa_circ_0010148 & -6.072531801 & $2.51 \times 10^{-2}$ & NM_198546 & SPATA21 & Down \\
\hline hsa_circ_0026524 & -6.476462428 & $1.13 \times 10^{-2}$ & NM_001417 & EIF4B & Down \\
\hline hsa_circ_0001890 & -7.728346085 & $6.81 \times 10^{-3}$ & NM_001006617 & MAPKAP1 & Down \\
\hline hsa_circ_0014349 & -9.677465499 & $4.32 \times 10^{-2}$ & NM_002870 & RAB13 & Down \\
\hline hsa_circ_0022807 & -15.16379666 & $4.45 \times 10^{-13}$ & NM_002689 & POLA2 & Down \\
\hline
\end{tabular}

MPP, M. pneumoniae pneumonia; NRMPP, non-refractory MPP; RMPP, refractory MPP; circRNA, circular RNA.

with pathogenicity (31). Upregulation of PTGS2 promotes inflammation, which may indicate that more severe inflammation was observed in the RMPP group in the present study (32). IL-8 is a chemotactic factor that attracts neutrophils, basophils and $\mathrm{T}$ cells, and it is also involved in neutrophil activation (33). FOSL1 encodes the regulator protein and is involved in cell proliferation, differentiation and transformation (34). The expression of PTGS2, IL- 8 and FOSL1 was significantly higher in the RMPP group compared with the NRMPP group in the present study, indicating that the upregulation of these proteins may participate in the pathogenicity of RMPP. In addition, RMPP cases exhibit a high activation level of the IL-17 signaling pathway, which may cause an autoimmune response and disease aggravation (35). Immunoglobulin heavy variable (IGHV)3-30, IGHV3-64D and IGHV5-10-1 belong to the V region of the variable domain of immunoglobulin heavy chains that participate in antigen recognition (36). In the present study, the disappearance of IGHV3-64D and IGHV5-10-1 genes and low expression of the IGHV3-30 gene in the RMPP group may be an important mechanism that leads to RMPP cases due to antigen recognition problems. However, further experiments are required to confirm these hypotheses.

In the present circRNA/mRNA analysis, circRNA function was found to be associated with the known function of the host linear transcripts. Based on the circRNA/miRNA/mRNA analysis conducted in the present study, several differentially expressed mRNAs were identified to be associated with the differentially expressed circRNAs. A total of 11 circRNAs were identified as the most significantly differentially expressed circRNAs between the HC and MPP groups. Among those, hsa_circ_0019868 [SH3 and PX domain-containing protein 2A (SH3PXD2A) gene] may be associated with MPP pathogenesis. SH3PXD2A is an adapter protein involved in the invasiveness of cancer cells that mediates the neurotoxic effect of the amyloid- $\beta$ peptide (37). The higher expression in MPP groups may enhance the invasiveness of M.pneumoniae. A total of 24 circRNAs were identified as the most significantly differentially expressed circRNAs between the NRMPP and RMPP groups. Among their target genes, hsa_circ_0001890 [target of rapamycin complex 2 subunit MAPKAP1 (MAPKAP1) gene], hsa_circ_0026524 (eukaryotic translation initiation factor 4B gene), hsa circ_0021640 [caprin-1 (CAPRIN1) gene], hsa_circ_0003781 [protein arginine N-methyltransferase 2 (PRMT2) gene], hsa_circ_0010131 [ephrin type-A receptor 2 (EPHA2) gene], hsa_circ_0025209 [NOP2 nucleolar protein (NOP2) gene] and hsa_circ_0023925 [phosphatidylinositol-binding clathrin assembly protein (PICALM) gene] may be associated with 
the pathogenesis of RMPP. The identified genes display the following characteristics: MAPKAP1 is involved in ciliogenesis, regulates cell proliferation and survival (38), and may serve as a novel anti-infection and antifibrogenesis genomic locus in chronic schistosomiasis japonica (39); NOP2 is involved in a ribosomal large subunit assembly and regulates cell proliferation (40); PRMT2 is involved in the regulation of proliferation and promotes apoptosis (41); EPHA2 regulates migration, integrin-mediated adhesion, proliferation and differentiation of cells (42); CAPRIN1 may regulate cell proliferation and migration in multiple cell types (43); and PICALM serves an important role in several processes, such as internalization of cell receptors, synaptic transmission and removal of apoptotic cells (44). Therefore, these genes might be involved in the pathogenesis of RMPP, but further investigations are required. In addition, the selected circRNAs (hsa_circ_0022808, hsa_circ_0006793, hsa_circ_0014390, hsa_circ_0014305 and hsa_circ_00216400) may represent valuable markers for the diagnosis of patients with early-stage RMPP and NRMPP. However, there were limited samples used in the present study; therefore, future studies should use larger sample sizes.

To conclude, the present study provided a comprehensive analysis of the expression levels of different lncRNAs, mRNAs and circRNAs between NRMPP and RMPP cases using rRNA-depleted RNA-sequencing techniques. The selected genes or circRNAs may aid with identifying the complex pathogenesis of RMPP and determining the diagnostic and therapeutic value of circRNAs in RMPP.

\section{Acknowledgements}

Not applicable.

\section{Funding}

This study was supported by the National Natural Science Foundation of China (grant nos. 81701990 and 81802817) and the National Science and Technology Major Project (grant no. 2018ZX10101004003001).

\section{Availability of data and materials}

The datasets used and/or analyzed during the current study are available from the corresponding author on reasonable request. The datasets generated and/or analyzed during the current study are available in the Sequence Read Archive repository (BioProject accession no. PRJNA704769; http://www.ncbi. nlm.nih.gov/bioproject/704769).

\section{Authors' contributions}

FH and GL made substantial contributions to conception and design. FH and GL confirm the authenticity of all the raw data. GL supervised the study. HF, DZ and DY provided the study materials. FH, HF, DY and TS collected and assembled the data. FH, HF, DZ and JZ analyzed and interpreted the data. FH, HF, DZ, TS and GL wrote and gave final approval of the manuscript. All authors read and approved the final manuscript.

\section{Ethics approval and consent to participate}

The present study was approved by the Ethics Committee at Guangzhou Women and Children's Medical Center (Guangzhou, China; approval no. 202009600). All the parents or legal guardians of the patients signed written informed consent forms and agreed to its content.

\section{Patient consent for publication}

All the parents or legal guardians of the patients signed written informed consent forms for the publication of patient data and associated images.

\section{Competing interests}

The authors declare that they have no competing interests.

\section{References}

1. Kumar S: Mycoplasma pneumoniae: A significant but underrated pathogen in paediatric community-acquired lower respiratory tract infections. Indian J Med Res 147: 23-31, 2018.

2. Medjo B, Atanaskovic-Markovic M, Radic S, Nikolic D, Lukac M and Djukic S: Mycoplasma pneumoniae as a causative agent of community-acquired pneumonia in children: Clinical features and laboratory diagnosis. Ital J Pediatr 40: 104, 2014.

3. Yang HJ, Song DJ and Shim JY: Mechanism of resistance acquisition and treatment of macrolide-resistant Mycoplasma pneumoniae pneumonia in children. Korean J Pediatr 60: 167-174, 2017.

4. Youn YS and Lee KY: Mycoplasma pneumoniae pneumonia in children. Clin Exp Pediatr 55: 42-47, 2012.

5. Waites KB, Xiao L, Liu Y, Balish MF and Atkinson TP: Mycoplasma pneumoniae from the respiratory tract and beyond. Clin Microbiol Rev 30: 747-809, 2017.

6. Rogozinski LE, Alverson BK and Biondi EA: Diagnosis and treatment of Mycoplasma pneumoniae in children. Minerva Pediatrica 69: 156-160, 2017.

7. Izumikawa K: Clinical features of severe or fatal Mycoplasma pneumoniae pneumonia. Front Microbiol 7: 800, 2016.

8. Shin JE, Cheon BR, Shim JW, Kim DS, Jung HL, Park MS and Shim JY: Increased risk of refractory Mycoplasma pneumoniae pneumonia in children with atopic sensitization and asthma. Korean J Pediatr 57: 271-277, 2014.

9. Bajantri B, Venkatram $S$ and Diaz-Fuentes G: Mycoplasma pneumoniae: A potentially severe infection. J Clin Med Res 10: 535-544, 2018.

10. Morozumi M, Hasegawa K, Kobayashi R, Inoue N, Iwata S, Kuroki H, Kawamura N, Nakayama E, Tajima T, Shimizu K and Ubukata K: Emergence of macrolide-resistant Mycoplasma pneumoniae with a 23S rRNA gene mutation. Antimicrob Agents Chemother 49: 2302-2306, 2005.

11. Matsuoka M, Narita M, Okazaki N, Ohya H, Yamazaki T, Ouchi K, Suzuki I, Andoh T, Kenri T, Sasaki Y, et al: Characterization and molecular analysis of macrolide-resistant Mycoplasma pneumoniae clinical isolates obtained in Japan. Antimicrob Agents Chemother 48: 4624-4630, 2004.

12. Oishi T, Narita M, Matsui K, Shirai T, Matsuo M, Negishi J, Kaneko T, Tsukano S, Taguchi T and Uchiyama M: Clinical implications of interleukin-18 levels in pediatric patients with Mycoplasma pneumoniae pneumonia. J Infect Chemother 17: 803-806, 2011.

13. Zhou YJ, Wang J, Chen WJ, Shen N, Tao Y, Zhao RK, Luo LJ, $\mathrm{Li} \mathrm{BR}$ and $\mathrm{Cao} \mathrm{Q}$ : Impact of viral coinfection and macrolide-resistant mycoplasma infection in children with refractory Mycoplasma pneumoniae pneumonia. BMC Infect Dis 20: 633, 2020.

14. Li MS, Liu YL, Zhang XL, Liu J and Wang P: Transcriptomic analysis of high-throughput sequencing about circRNA, lncRNA and mRNA in bladder cancer. Gene 677: 189-197, 2018. 
15. Beermann J, Piccoli MT, Viereck J and Thum T: Non-coding RNAs in development and disease: Background, mechanisms, and therapeutic approaches. Physiol Rev 96: 1297-1325, 2016.

16. Meng S, Zhou H, Feng Z, Xu Z, Tang Y, Li P and Wu M: CircRNA: Functions and properties of a novel potential biomarker for cancer. Mol Cancer 16: 94, 2017.

17. Zhang HD, Jiang LH, Sun DW, Hou JC and Ji ZL: CircRNA: A novel type of biomarker for cancer. Breast Cancer 25: 1-7, 2018.

18. Wu H, Wu S, Zhu Y, Ye M, Shen J, Liu Y, Zhang YS and Bu S: Hsa_circRNA_0054633 is highly expressed in gestational diabetes mellitus and closely related to glycosylation index. Clin Epigenetics 11: 22, 2019.

19. Cui X, Niu W, Kong L, He M, Jiang K, Chen S, Zhong A, Li W, Lu J and Zhang L: hsa_circRNA_103636: Potential novel diagnostic and therapeutic biomarker in major depressive disorder. Biomarkers Med 10: 943-952, 2016.

20. Lee YC, Chang CH, Lee WJ, Liu TY, Tsai CM, Tsai TA, Tsai CK, Kuo KC, Chen CC, Niu CK and Yu HR: Altered chemokine profile in Refractory Mycoplasma pneumoniae pneumonia infected children. J Microbiol Immunol Infect: S1684-S1182(20)30090-30096, 2020.

21. Kim D, Langmead B and Salzberg S: HISAT: A fast spliced aligner with low memory requirements. Nat Methods 12: 357-360, 2015

22. Anders S, Pyl PT and Huber W: HTSeq-a Python framework to work with high-throughput sequencing data. Bioinformatics 31 : $166-169,2015$

23. Love MI, Huber W and Anders S: Moderated estimation of fold change and dispersion for RNA-seq data with DESeq2. Genome Biol 15: 550, 2014.

24. Memczak S, Jens M, Elefsinioti A, Torti F, Krueger J, Rybak A Maier L, Mackowiak SD, Gregersen LH, Munschauer M, et al: Circular RNAs are a large class of animal RNAs with regulatory potency. Nature 495: 333-338, 2013.

25. Gao Y, Wang J and Zhao F: CIRI: An efficient and unbiased algorithm for de novo circular RNA identification. Genome Biol 16: 4, 2015.

26. Young MD, Wakefield MJ, Smyth GK and Oshlack A: Gene ontology analysis for RNA-seq: Accounting for selection bias. Genome Biol 11: R14, 2010.

27. Kanehisa M, Araki M, Goto S, Hattori M, Hirakawa M, Itoh M, Katayama T, Kawashima S, Okuda S, Tokimatsu T and Yamanishi Y: KEGG for linking genomes to life and the environment. Nucleic Acids Res 36: D480-D484, 2008.

28. Xie C, Mao X, Huang J, Ding Y, Wu J, Dong S, Kong L, Gao G Li CY and Wei L: KOBAS 2.0: A web server for annotation and identification of enriched pathways and diseases. Nucleic Acids Res 39:W316-W322, 2011.

29. Livak KJ and Schmittgen TD: Analysis of relative gene expression data using real-time quantitative PCR and the 2(-Delta Delta C(T)) method. Methods 25: 402-408, 2001.

30. Liu S, Xie X, Lei H, Zou B and Xie L: Identification of key circRNAs/lncRNAs/miRNAs/mRNAs and pathways in preeclampsia using bioinformatics analysis. Med Sci Monit 25: $1679-1693,2019$.
31. Chaudhry R, Ghosh A and Chandolia A: Pathogenesis of Mycoplasma pneumoniae: An update. Indian J Med Microbiol 34: 7-16, 2016.

32. Rumzhum NN and Ammit AJ: Cyclooxygenase 2: Its regulation, role and impact in airway inflammation. Clin Exp Allergy 46: 397-410, 2016.

33. Cavallaro EC, Liang KK, Lawrence MD, Forsyth KD and Dixon DL: Neutrophil infiltration and activation in bronchiolitic airways are independent of viral etiology. Pediatr Pulmonol 52: 238-246, 2017.

34. Maurus K, Hufnagel A, Geiger F, Graf S, Berking C, Heinemann A, Paschen A, Kneitz S, Stigloher C, Geissinger E, et al: The AP-1 transcription factor FOSL1 causes melanocyte reprogramming and transformation. Oncogene 36: 5110-5121, 2017.

35. Hu Y, Shen F, Crellin NK and Ouyang W: The IL-17 pathway as a major therapeutic target in autoimmune diseases. Ann N Y Acad Sci 1217: 60-76, 2011.

36. McHeyzer-Williams M, Okitsu S, Wang N and McHeyzerWilliams L: Molecular programming of B cell memory. Nature Rev Immunol 12: 24-34, 2011.

37. Chen Q, Jiang S, Liu H, Gao Y, Yang X, Ren Z, Gao Y, Xiao L, $\mathrm{Hu} \mathrm{H}, \mathrm{Yu} \mathrm{Y}$, et al: Association of IncRNA SH3PXD2A-AS1 with preeclampsia and its function in invasion and migration of placental trophoblast cells. Cell Death Dis 11: 583, 2020.

38. Cardenas-Rodriguez M, Irigoín F, Osborn DP, Gascue C, Katsanis N, Beales PL and Badano JL: The Bardet-Biedl syndrome-related protein CCDC28B modulates mTORC2 function and interacts with SIN1 to control cilia length independently of the mTOR complex. Hum Mol Genet 22: 4031-4042, 2013.

39. Zhu X, Zhang J, Fan W, Gong Y, Yan J, Yuan Z, Wu L, Cui H, Luo H, Kong Q, et al: MAPKAP1 rs10118570 polymorphism is associated with anti-infection and anti-hepatic fibrogenesis in schistosomiasis japonica. PLoS One 9: e105995, 2014

40. Sloan KE, Bohnsack MT and Watkins NJ: The 5S RNP couples p53 homeostasis to ribosome biogenesis and nucleolar stress. Cell Rep 5: 237-247, 2013.

41. Zhong J, Chen YJ, Chen L, Shen YY, Zhang QH, Yang J, Cao RX, Zu XY and Wen GB: PRMT2 $\beta$, a C-terminal splice variant of PRMT2, inhibits the growth of breast cancer cells. Oncol Rep 38: 1303-1311, 2017.

42. Finney AC, Funk SD, Green JM, Yurdagul A Jr, Rana MA, Pistorius R, Henry M, Yurochko A, Pattillo CB, Traylor JG, et al: EphA2 expression regulates inflammation and fibroproliferative remodeling in atherosclerosis. Circulation 136: 566-582, 2017.

43. Li XQ, Song JY, Lv W, Zhang D and Wu JZ: Circular circ_0000885 promotes hepatocellular carcinoma proliferation by epigenetically upregulating caprin1. Eur Rev Med Pharmacol Sci 23: 7848-7854, 2019.

44. Miller SE, Sahlender DA, Graham SC, Höning S, Robinson MS, Peden AA and Owen DJ: The molecular basis for the endocytosis of small R-SNAREs by the clathrin adaptor CALM. Cell 147: 1118-1131, 2011.

(i) $($ ) This work is licensed under a Creative Commons Attribution-NonCommercial-NoDerivatives 4.0 International (CC BY-NC-ND 4.0) License. 